\title{
Une nouvelle approche dans la théorie des entiers friables*
}

\author{
R. de la Bretèche \& G. Tenenbaum
}

\begin{abstract}
Using a new approach starting with a residue computation we sharpen some of the known estimates for the counting function of friable integers. The gained accuracy turns out to be crucial for various applications, some of which concern fundamental questions in probabilistic number theory.

Keywords: Friable integers, saddle-point method, Montgomery-Wirsing inequality, transfer principle, zero-free regions, exponential sums over primes.

Résumé. Grâce à une nouvelle approche, dont le point de départ est un calcul de résidu, nous précisons certaines des estimations connues pour la fonction de comptage des entiers friables. Le gain se révèle crucial pour diverses applications, dont certaines concernent des questions fondamentales de la théorie probabiliste des nombres.

Mots clefs : Entiers friables, méthode du col, inégalité de Montgomery-Wirsing, principe de transfert, région sans zéro, sommes d'exponentielles sur les nombres premiers.
\end{abstract}

\section{Introduction}

Désignons par $P^{+}(n)$ le plus grand facteur premier d'un entier naturel générique $n$, avec la convention $P^{+}(1)=1$. Étant donné un paramètre $y \geqslant 2$, on dit qu'un nombre $n$ est $y$-friable s'il vérifie $P^{+}(n) \leqslant y$. L'étude des entiers friables occupe depuis une trentaine d'années une place privilégiée dans le développement de la théorie analytique et probabiliste des nombres. Le lien direct avec l'hypothèse de Riemann est explicité dans [11]. On trouvera, entre autres, dans les survols [14], [17], [9], dans les actes de colloque [7] et dans la monographie [19] une liste non exhaustive des motivations de cet engouement et une description de nombreux résultats fondamentaux de la théorie.

Notons classiquement $S(x, y)$ l'ensemble des entiers $y$-friables n'excédant pas $x$ et $\Psi(x, y)$ son cardinal. Amorcée par Dickman en 1930, l'étude asymptotique de $\Psi(x, y)$ a été abordée via diverses méthodes. Pour les grandes valeurs de $y$, la voie des équations fonctionnelles a été initialement suivie, notamment par de Bruijn [5], [6], et Hildebrand [12]. Pour les petites valeurs de $y$, la méthode géométrique de comptage de points dans un domaine limité par des hyperplans a été explorée par Ennola [8], qui met également en œuvre une technique d'inversion de Laplace effective. Enfin, la méthode du col élaborée par Hildebrand et Tenenbaum [13] fournit une évaluation universelle dont sont tirées les meilleures estimations connues à ce jour — voir en particulier le chapitre III.5 de [19].

Le comportement asymptotique de $\Psi(x, y)$ présente un changement de phase autour de la valeur $y=\log x$. Ce phénomène classique trouve son origine dans le fait que le produit des nombres premiers n'excédant pas $y$ devient notablement plus petit que $x$ dès $y \leqslant c \log x$ où $c$ est une constante $<1$ : cela impose aux entiers $y$-friables de l'ordre de $x$ des valuations $p$-adiques élevées et partant réduit le nombre de possibilités.

Alors que les termes d'erreur disponibles sont globalement satisfaisants pour $y>\log x$, et même pour $y>(\log x)^{1+o(1)}$, ceux qui relèvent de valeurs plus faibles de $y$ se révèlent souvent inopérants pour les applications. C'est notamment le cas pour l'inégalité de TuránKubilius friable - voir infra.

Nous nous proposons dans ce travail d'explorer, dans la zone située en dessous du seuil, les possibilités d'évaluation de $\Psi(x, y)$ par une combinaison de la méthode des

\footnotetext{
* Nous incluons ici quelques corrections relativement à la version publiée.
} 
résidus, d'estimations de type point-selle, de majorations de sommes d'exponentielles sur les nombres premiers, et d'un principe de transfert, dû à Wirsing et Montgomery, pour les moyennes quadratiques de séries de Dirichlet. Nous obtenons ainsi des formules asymptotiques significativement plus précises que celles de la littérature et dont les conséquences, notamment au comportement local — voir en particulier les Corollaires 3.1, 3.2 et 3.3 infra - , sont suffisantes pour la quasi-totalité des applications envisagées ; certaines sont décrites plus loin.

L'adjonction des estimations issues de la méthode du col à celles du présent travail finalise l'étude globale de la fonction de comptage $\Psi(x, y)$. Un trait spécifique distinguant nos résultats des évaluations antérieures tient à la nature des termes d'erreur, décroissants à la fois en $x$ et en $y$. Pour les très petites valeurs du paramètre de friabilité, la précision est de l'ordre d'une puissance fractionnaire du terme principal.

L'étude qui suit est donc restreinte au domaine

$$
2 \leqslant y \leqslant(\log x)^{1-\varepsilon},
$$

où $\varepsilon>0$ désigne une constante arbitraire.

\section{2. Énoncé des estimations principales}

Soit

$$
\zeta(s, y):=\prod_{p \leqslant y}\left(1-\frac{1}{p^{s}}\right)^{-1} \quad(s \in \mathbb{C}, y \geqslant 2) .
$$

Ici et dans la suite, nous notons systématiquement $s=\sigma+i \tau$; la lettre $p$ est réservée pour désigner un nombre premier, et nous dénotons par $\mathbb{P}$ l'ensemble de tous les nombres premiers.

Pour $x \in \mathbb{R}^{+} \backslash \mathbb{N}$, nous avons

$$
\Psi(x, y)=\frac{1}{2 \pi i} \int_{\alpha-i \infty}^{\alpha+i \infty} \frac{\zeta(s, y) x^{s}}{s} \mathrm{~d} s,
$$

où $\alpha=\alpha(x, y)$ dénote le point-selle réel de $\zeta(s, y) x^{s}$, défini par l'équation

$$
\sum_{p \leqslant y} \frac{\log p}{p^{\alpha}-1}=\log x \text {. }
$$

Il est établi dans [13] que

$$
\alpha=\frac{1}{\log y} \log \left(1+\frac{y}{\log x}\right)\left\{1+O\left(\frac{\log _{2} 2 y}{\log y}\right)\right\} \quad(x \geqslant y \geqslant 2),
$$

et, précisément, que, pour tout $\varepsilon>0$,

$$
\alpha= \begin{cases}1-\frac{\xi(u)}{\log y}+O\left(\frac{1}{L_{\varepsilon}(y)}+\frac{1}{u(\log y)^{2}}\right) & \left((\log x)^{1+\varepsilon} \leqslant y \leqslant x\right), \\ \frac{\log (1+y / \log x)}{\log y}\left\{1+O\left(\frac{1}{\log y}\right)\right\} & \left(2 \leqslant y \leqslant(\log x)^{3}\right),\end{cases}
$$

où l'on a posé $L_{\varepsilon}(y):=\exp \left\{(\log y)^{3 / 5-\varepsilon}\right\}$ et où $\xi(v)$ est défini comme la solution de l'équation $\mathrm{e}^{\xi(v)}=1+v \xi(v)$ pour $v>1$ et $\xi(1):=0$.

Ainsi que l'a souligné de Bruijn dans [5], il est raisonnable d'attendre que le résidu à l'origine, noté $P_{y}(\log x)$, fournisse une bonne approximation du membre de gauche lorsque $y$ est petit devant $x$. Un tel résultat a été obtenu par Ennola [8] dans le domaine $2 \leqslant y \leqslant(\log x)^{3 / 4-\varepsilon}$ avec un terme d'erreur relatif $1+O\left(1 /(\log x)^{1 / 8-\varepsilon}\right)$, améliorable en

$$
1+O\left(\frac{y^{2}}{(\log x) \log y}\right)
$$

lorsque $y \leqslant(\log x)^{7 / 16}$. 
Nous établissons effectivement que $P_{y}(\log x)$ est une bonne approximation de $\Psi(x, y)$ dans l'ensemble du domaine (1.1) avec un terme d'erreur significativement plus précis et globalement compatible avec les applications envisagées.

La fonction génératrice $x^{s} \zeta(s, y) / s$ possède une infinité d'autres pôles, aux points $2 \pi i k / \log p$, pour $p \leqslant y, k \in \mathbb{Z}^{*}$. En raison du théorème fondamental de l'arithmétique, ces pôles sont simples : cela explique en partie le fait que les résidus correspondants n'ont pas une influence essentielle sur le comportement asymptotique de $\Psi(x, y)$ et constituent, dans le domaine $(1 \cdot 1)$, des termes secondaires par rapport au résidu à l'origine. Alors que la prise en compte de tous les résidus fournit évidemment une formule exacte pour $\Psi(x, y)$, on peut imaginer qu'une prise en compte partielle permettrait d'élucider le changement de phase autour de $y=\log x$. Une telle démarche serait certainement délicate en raison de la semi-convergence des séries des résidus, elle-même liée à l'indépendance sur $\mathbb{Q}$ des $\log p$ pour $p \leqslant y$. On a par exemple, notant $\langle v\rangle$ la partie fractionnaire d'un nombre réel $v$,

$$
\Psi(x, 2)-P_{2}(\log x)=\frac{1}{2}-\left\langle\frac{\log x}{\log 2}\right\rangle=\lim _{K \rightarrow \infty} \sum_{0<|k| \leqslant K} \frac{x^{2 \pi i k / \log 2}}{2 \pi k i} .
$$

Commençons par expliciter $P_{y}(\log x)$. Notant $\left\{B_{m}\right\}_{m=0}^{\infty}$ la suite des nombres de Bernoulli, nous avons le développement de Laurent

$$
\frac{1}{1-\mathrm{e}^{-s}}=\sum_{m \geqslant 0} \frac{(-1)^{m} B_{m}}{m !} s^{m-1} \quad(|s|<2 \pi),
$$

d'où

$$
\zeta(s, y)=\sum_{n \geqslant 0}(-1)^{n} s^{n-\pi(y)} \sum_{* n} \prod_{p \leqslant y} \frac{B_{j_{p}}(\log p)^{j_{p}-1}}{j_{p} !} \quad(|s|<2 \pi / \log y),
$$

où le symbole $* n$ signifie que la sommation porte sur toutes les suites $\left\{j_{p}\right\}_{p \leqslant y} \in \mathbb{N}^{\pi(y)}$ telles que $\sum_{p \leqslant y} j_{p}=n .^{(1)}$ Il s'ensuit que

$$
P_{y}(\xi)=\sum_{0 \leqslant k \leqslant \pi(y)} \frac{\xi^{\pi(y)-k} c_{k}(y)}{(\pi(y)-k) ! \prod_{p \leqslant y} \log p}
$$

avec

$$
c_{k}(y):=(-1)^{k} \sum_{* k} \prod_{p \leqslant y} \frac{B_{j_{p}}(\log p)^{j_{p}}}{j_{p} !} \quad(k \geqslant 0) .
$$

Nous pouvons énoncer le résultat suivant, où nous faisons usage de la notation

$$
Y_{\varepsilon}:=\mathrm{e}^{(\log y)^{3 / 2-\varepsilon}} .
$$

Théorème 2.1. Pour chaque $\varepsilon>0$ et sous la condition (1·1), nous avons

$$
\Psi(x, y)=\left\{1+O\left(\frac{1}{Y_{\varepsilon} \log x}\right)\right\} P_{y}(\log x) .
$$

1. Ainsi qu'il est d'usage dans la littérature en langue française, l'ensemble $\mathbb{N}$ désigne ici l'ensemble des entiers naturels positifs ou nuls. 
Remarque. Nous donnons au Théorème 2.4 une évaluation asymptotique pour les coefficients $c_{k}(y)$ et explicitons les premières valeurs au Lemme 5.1. La majoration issue de l'estimation établie en $(2 \cdot 22)$ infra

$$
c_{k}(y)=\frac{\vartheta(y)^{k}}{2^{k} k !}\left\{1+O\left(\frac{k^{2}}{\pi(y)}\right)\right\} \quad(0 \leqslant k \leqslant \pi(y)),
$$

où $\vartheta$ désigne la fonction de Tchébychev, permet de retrouver le théorème 1 d'Ennola [8], valide pour

$$
2 \leqslant y \leqslant c \sqrt{\log x \log _{2} x}
$$

où $c>0$ est une constante assez petite. Nous verrons au Théorème 2.4 que ce domaine peut être étendu.

Pour $\sigma:=\Re e s>0$, posons

$$
\begin{aligned}
\varphi_{0}(s, y) & :=\log \zeta(s, y), \quad \varphi_{k}(s, y):=\varphi_{0}^{(k)}(s, y), \\
\sigma_{k} & =\sigma_{k}(\alpha, y):=(-1)^{k} \varphi_{k}(\alpha, y) \quad(k \in \mathbb{N}),
\end{aligned}
$$

où la branche du logarithme complexe est obtenue par addition des déterminations principales $\log \left\{1 /\left(1-p^{-s}\right)\right\}$ pour $p \leqslant y$. Notant

$$
M(x, y):=\frac{x^{\alpha} \zeta(\alpha, y)}{\alpha \sqrt{2 \pi \sigma_{2}}},
$$

où $\alpha$ est défini comme en $(2 \cdot 1)$, Hildebrand et Tenenbaum [13] ont établi par la méthode du col que l'estimation

$$
\Psi(x, y)=M(x, y)\left\{1+O\left(\frac{1}{\pi(y)}\right)\right\}
$$

est valable dans le domaine $y \leqslant \log x$. Il résulte donc du Théorème 2.1 que l'on a, dans le domaine $(1 \cdot 1)$,

$$
P_{y}(\log x)=M(x, y)\left\{1+O\left(\frac{1}{\pi(y)}\right)\right\} .
$$

Une étude directe permet d'affiner cette estimation. Nous posons

$$
\mathfrak{s}(h):=h ! \mathrm{e}^{h} h^{-h} / \sqrt{2 \pi h} \quad(h \geqslant 1),
$$

de sorte que la formule de Stirling implique $\mathfrak{s}(h)=1+1 /(12 h)+O\left(1 / h^{2}\right)$ pour $h \geqslant 1$.

Proposition 2.2. Soit $\varepsilon>0$. Sous la condition (1.1) et en notant $h:=\pi(y)$, nous avons

$$
\begin{aligned}
P_{y}(\log x) & =\left\{1+O\left(\frac{h y^{2}}{(\log x)^{2}}\right)\right\} \frac{M(x, y)}{\mathfrak{s}(h)} \\
& =\left\{1-\frac{1}{12 h}+O\left(\frac{1}{h^{2}}+\frac{h y^{2}}{(\log x)^{2}}\right)\right\} M(x, y) .
\end{aligned}
$$

Remarques. (i) Dans cet énoncé et les suivants, nous adoptons la convention standard selon laquelle les constantes implicites peuvent dépendre des constantes fixées initialement.

(ii) Ce résultat implique que le terme d'erreur de (2.13) est optimal sous la condition $y \leqslant c \sqrt{\log x \log _{2} x}$ où $c$ est une constante assez petite. 
(iii) Le biais, mis en évidence par (2·15), des évaluations asymptotiques de $\Psi(x, y)$ obtenues par la méthode du col pour les petites valeurs du paramètre $y$ s'explique par la nature de la singularité à l'origine de la fonction $\zeta(s, y)$, qui est du type $1 / s^{h}$ avec $h=\pi(y)$. Nous avons en effet

$$
\frac{1}{2 \pi i} \int_{\alpha-i \infty}^{\alpha+i \infty} \frac{x^{s}}{s^{h+1}} \mathrm{~d} s=\frac{(\log x)^{h}}{h !}
$$

pour tout $\alpha>0$. Or, le col relatif à la fonction $x^{s} / s^{h}$ vaut $\alpha_{h}:=h / \log x$. Le terme principal issu d'un emploi standard de la méthode du col vaut donc

$$
\frac{1}{2 \pi} \int_{-\infty}^{\infty} \frac{x^{\alpha_{h}}}{\alpha_{h}^{h+1}} \mathrm{e}^{-\tau^{2} \sigma_{h} / 2} \mathrm{~d} \tau=\frac{x^{\alpha_{h}}}{\alpha_{h}^{h+1} \sqrt{2 \pi \sigma_{h}}}=\frac{(\log x)^{h}}{h !} \mathfrak{s}(h),
$$

où nous avons posé $\sigma_{h}=h / \alpha_{h}^{2}$.

La méthode des résidus employée pour établir le Théorème 2.1 fonctionne également pour estimer les quantités

$$
\Psi_{m}(x, y):=\sum_{\substack{n \in S(x, y) \\(n, m)=1}} 1
$$

lorsque l'entier $m$ ne possède pas un trop grand nombre de facteurs premiers. Nous nous restreignons ici au cas où le nombre $\omega(m)$ des facteurs premiers distincts de $m$ est borné, ce qui est suffisant pour certaines applications fondamentales, notamment l'inégalité de Turán-Kubilius friable [4]. Nous posons

$$
g_{m}(s):=\prod_{p \mid m}\left(1-p^{-s}\right) \quad\left(m \in \mathbb{N}^{*}, s \in \mathbb{C}\right)
$$

et notons $\zeta_{m}(s ; y)=g_{m}(s) \zeta(s ; y)$ la série de Dirichlet associée à la fonction de comptage $\Psi_{m}(x, y)$ lorsque $P^{+}(m) \leqslant y$.

Théorème 2.3. Soient $\varepsilon>0, M \geqslant 1$ fixés. Sous les conditions (1.1), $m \geqslant 1$, $0 \leqslant \omega(m) \leqslant M, P^{+}(m) \leqslant y$, nous avons uniformément

$$
\Psi_{m}(x, y)=\left\{1+O\left(\frac{1}{Y_{\varepsilon} \log x}\right)\right\} P_{y, m}(\log x),
$$

où l'on a posé

$$
P_{y, m}(\xi):=\sum_{0 \leqslant j \leqslant \pi(y)}(-1)^{j} \frac{\lambda_{j}(m)}{j !} P_{y}^{(j)}(\xi)
$$

avec $\lambda_{j}(m):=\sum_{d \mid m} \mu(d)(\log d)^{j}(0 \leqslant j \leqslant \pi(y))$.

Nous avons également

$$
P_{y, m}(\xi)=\sum_{d \mid m} \mu(d) P_{y}(\xi-\log d)=\sum_{0 \leqslant k \leqslant \pi(y)-\omega(m)} \frac{\xi^{\pi(y)-\omega(m)-k} c_{k}(y ; m)}{(\pi(y)-\omega(m)-k) ! \prod_{\substack{p \leqslant y \\ p \nmid m}} \log p}
$$

où l'on a posé

$$
c_{k}(y ; m):=(-1)^{k} \sum_{* k} \prod_{\substack{p \leqslant y \\ p \nmid m}} \frac{B_{j_{p}}(\log p)^{j_{p}}}{j_{p} !} \quad(0 \leqslant k \leqslant \pi(y)-\omega(m)) .
$$


Remarques. (i) On vérifie facilement que $\lambda_{j}(m)=0$ si $j<\omega(m)$.

(ii) Un emploi direct de la méthode du col permet, dans les hypothèses du Théorème 2.3, d'obtenir l'évaluation

$$
\Psi_{m}(x, y) \asymp \frac{x^{\alpha} \zeta_{m}(\alpha, y)}{\sqrt{\pi(y)}} \asymp g_{m}(\alpha) \Psi(x, y) .
$$

L'énoncé suivant fournit une évaluation asymptotique des coefficients $c_{k}(y ; m)$ et une formule asymptotique pour le terme principal de $(2 \cdot 16)$ dans le sous-domaine

$$
y=o\left((\log x)^{2 / 3}\left(\log _{2} x\right)^{1 / 3}\right) .
$$

Nous posons

$$
\vartheta_{r}(y ; m)=\sum_{\substack{p \leqslant y \\ p \nmid m}}(\log p)^{r} \quad(r \geqslant 0, m \geqslant 1),
$$

de sorte que $\vartheta_{0}(y ; m)=\pi(y)-\omega(m)$, et notons simplement $\vartheta_{r}(y):=\vartheta_{r}(y ; 1)(y \geqslant 2)$.

Théorème 2.4. Soit $M \geqslant 1$. Sous les conditions $m \geqslant 1,0 \leqslant \omega(m) \leqslant M, P^{+}(m) \leqslant y$, et en notant $h:=\pi(y)$, nous avons uniformément

$$
c_{k}(y ; m)=\frac{\vartheta_{1}(y ; m)^{k}}{2^{k} k !}\left\{1+O\left(\frac{k(k-1)}{h}\right)\right\} \quad(k \leqslant h-\omega(m)) .
$$

En particulier, sous les mêmes conditions en $y$ et $m$, nous avons

$$
P_{y, m}(\xi)=\frac{\left\{\xi+\frac{1}{2} \vartheta_{1}(y ; m)\right\}^{h-\omega(m)}}{\{h-\omega(m)\} ! \prod_{\substack{p \leqslant y \\ p \nmid m}} \log p}\left\{1+O\left(\frac{y^{2} h}{\xi^{2}}\right)\right\} \quad(\xi \geqslant 1) .
$$

Remarque. Alors qu'Ennola [8] a élucidé le comportement asymptotique de $P_{y, m}(\log x)$ lorsque $y=o\left(\sqrt{\log x \log _{2} x}\right)$, la formule $(2 \cdot 23)$ fournit un équivalent dans la région $(2 \cdot 21)$.

Un développement plus précis des coefficients $c_{k}(y ; m)$ permettrait d'agrandir encore ce domaine. On peut ainsi établir, par exemple, que l'on a, pour $y \geqslant 2, \xi \geqslant 1$,

$$
P_{y}(\xi)=\frac{\left\{\xi+\frac{1}{2} \vartheta_{1}(y)\right\}^{h}}{h ! \prod_{p \leqslant y} \log p}\left\{1-\frac{h(h-1) \vartheta_{2}(y)}{6 \xi^{2}}+O\left(\frac{y^{3} h}{\xi^{3}}+\frac{y^{4} h^{2}}{\xi^{4}}\right)\right\} .
$$

\section{Conséquences}

Nous conservons la notation $h=\pi(y)$ dans toute la suite de ce travail.

Dans ce paragraphe, nous développons des répercussions directes de nos résultats sur les estimations relatives au comportement local des fonctions $\Psi_{m}(x, y)$.

Le corollaire suivant précise les discontinuités de $\Psi(x, y)$ par rapport à la seconde variable lorsque le paramètre de friabilité $y$ est petit. L'énoncé découle directement de $(2 \cdot 23)$ avec $m=1$.

Corollaire 3.1. Soit $\varepsilon>0$. Sous les conditions $(1 \cdot 1)$ et $y \in \mathbb{P}$, nous avons

$$
\frac{\Psi(x, y)}{\Psi(x, y-)}=\frac{\log x}{h \log y}\left\{1+\frac{\vartheta_{1}(y)+(h-1) \log y}{2 \log x}+O\left(\frac{1}{Y_{\varepsilon} \log x}+\frac{y^{2} h}{(\log x)^{2}}\right)\right\} .
$$


Remarques. (i) Le membre de droite de $(3 \cdot 1)$ est $\sim(\log x) / y$ dans le domaine $(2 \cdot 21)$.

(ii) Une formule analogue à $(3 \cdot 1)$ résulte des estimations d'Ennola [8] dans un domaine restreint. Cependant, le terme d'erreur, toujours $\gg\left(y^{2} / \log x \log y\right)$, inhérent à un tel résultat ne permet pas de faire apparaître la correction au premier ordre figurant dans l'accolade.

Le résultat suivant apporte une précision supplémentaire aux évaluations du théorème 2.4 de [1]. À fins de référence ultérieure, nous l'énonçons en toute généralité. Nous posons $\bar{u}:=\min (u, y / \log y), u_{y}:=\bar{u}+(\log y) / \log (u+2)$, et

$$
\mathfrak{R}:=\mathfrak{R}(d ; x, y):=\min \left\{\frac{1}{Y_{\varepsilon} \log x}+\frac{y^{2} h}{(\log x)^{2}}, \frac{1}{u_{y}}+\frac{\log d}{\log x}\right\} .
$$

Notons que, dans le domaine $y \leqslant \log x$, nous avons

$$
\mathfrak{R} \asymp \min \left\{\frac{1}{Y_{\varepsilon} \log x}+\frac{y^{2} h}{(\log x)^{2}}, \frac{\log y}{y}+\frac{\log d}{\log x}\right\} .
$$

Corollaire 3.2. Soit $\varepsilon>0$. Il existe des constantes absolues positives $b_{1}, b_{2}$, et une fonction $b=b(x, y ; d, m)$ satisfaisant à $b_{1} \leqslant b \leqslant b_{2}(x \geqslant y \geqslant 2, d \geqslant 1, m \geqslant 1)$ telles que, sous les conditions $x \geqslant y \geqslant 2,1 \leqslant d \leqslant x^{1-\varepsilon}, P^{+}(m) \leqslant y, \mu(m)^{2}=1$, et $\omega(m) \ll 1$, on ait uniformément

$$
\Psi_{m}\left(\frac{x}{d}, y\right)=\left(1-\frac{t^{2}}{u^{2}+\bar{u}^{2}}\right)^{b \bar{u}} \frac{h ! g_{m}(\alpha) \Psi(x, y)}{\{h-\omega(m)\} ! h^{\omega(m)} d^{\alpha}}\left\{1+\frac{\omega(m) \log m}{2 \log x}+O(\mathfrak{E})\right\}
$$

où l'on a posé $t:=(\log d) / \log y, \mathfrak{E}:=\omega(m) t / u+\mathfrak{R}$. De plus, quitte à ajouter $t / u$ au terme d'erreur, cette estimation demeure valide pour $1 \leqslant d \leqslant x$.

Nous explicitons ci-dessous une conséquence immédiate, utile pour les applications, $\mathrm{du}$ cas $d=1, \omega(m)=1$ ou 2 , de l'énoncé précédent.

Corollaire 3.3. Soit $\varepsilon>0$. Sous la condition (1.1) et pour tous nombres premiers distincts $p, q$ n'excédant pas $y$, nous avons

$$
\begin{aligned}
& \Psi_{p}(x, y)=g_{p}(\alpha) \Psi(x, y)\left\{1+\frac{\log p}{2 \log x}+O\left(\frac{y^{2} h}{(\log x)^{2}}+\frac{1}{Y_{\varepsilon} \log x}\right)\right\} \\
& \Psi_{p q}(x, y)=g_{p q}(\alpha)\left(1-\frac{1}{h}\right) \Psi(x, y)\left\{1+\frac{\log p q}{\log x}+O\left(\frac{y^{2} h}{(\log x)^{2}}+\frac{1}{Y_{\varepsilon} \log x}\right)\right\}
\end{aligned}
$$

Ce dernier résultat joue notamment un rôle crucial dans l'étude de l'inégalité de Turán-Kubilius friable telle qu'entreprise dans [2], et poursuivie dans [15], [10], [3]. Ce problème fondamental en théorie probabiliste des nombres est lié à la modélisation de la répartition, sur l'ensemble des entiers friables, d'une fonction arithmétique additive à valeurs complexes par une somme de variables aléatoires indépendantes. Plus précisément, étant donnés une fonction additive $f$ et un couple de nombres réels $(x, y)$ tel que $x \geqslant y \geqslant 2$, nous définissons des variables aléatoires géométriques $\xi_{p}$ par

$$
\mathbb{P}\left(\xi_{p}=f\left(p^{\nu}\right)\right)=\frac{1-1 / p^{\alpha}}{p^{\nu \alpha}} \quad(\nu=0,1,2, \ldots)
$$

avec la convention que, si plusieurs valeurs (en nombre éventuellement infini) $f\left(p^{\nu}\right)$ coïncident, la probabilité correspondante est obtenue en sommant les probabilités apparaissant au second membre. Notant

$$
Z_{f}=Z_{f, x, y}=\sum_{p \leqslant y} \xi_{p}
$$


où les $\xi_{p}$ sont indépendantes, et introduisant la variance semi-empirique

$$
V_{f}(x, y):=\frac{1}{\Psi(x, y)} \sum_{n \in S(x, y)}\left|f(n)-\mathbb{E}\left(Z_{f}\right)\right|^{2},
$$

le problème consiste alors à majorer la quantité

$$
C(x, y):=\sup _{f} V_{f}(x, y) / \mathbb{V}\left(Z_{f}\right) \quad(x \geqslant y \geqslant 2)
$$

où le supremum est pris sur l'ensemble des fonctions arithmétiques additives complexes.

Classiquement, le comportement asymptotique de $C(x, y)$ mesure l'écart entre la théorie probabiliste des nombres et celle des probabilités. À l'instar du cas historique $y=x$, le fait que $C(x, y)$ soit bornée met en évidence la pertinence globale du modèle probabiliste alors que la comparaison asymptotique de cette quantité à $1+o(1)$ souligne les limites d'une hypothèse d'indépendance.

À la suite des travaux précités, nous savons que

$$
\sup _{h(x) \leqslant y \leqslant x} C(x, y) \ll 1 \quad(x \geqslant 2)
$$

pour $h(x):=\sqrt{\log x} \log _{2} x$, tandis que l'indépendance asymptotique sous la forme $C(x, y)=1+o(1)$ a lieu dès que $y / \log x$ et $u:=(\log x) / \log y$ tendent vers l'infini.

Nous avons pu établir dans [4] la majoration universelle

$$
\sup _{x \geqslant y \geqslant 2} C(x, y) \ll 1
$$

et, grâce aux résultats présentés plus haut, obtenir le renseignement supplémentaire

$$
C_{1}(x, y) \leqslant 1+o(1) \quad(1 / u+1 / y \rightarrow 0)
$$

où $C_{1}(x, y)$ désigne la quantité analogue à $(3 \cdot 5)$ lorsque le supremum est pris sur l'ensemble des fonctions arithmétiques complexes fortement additives. Compte tenu des résultats de [2] mentionnés plus haut, $(3 \cdot 6)$ devient une égalité dès que $u$ et $y / \log x$ tendent vers l'infini. Nous renvoyons à [4] pour des développements et résultats complémentaires concernant cette question.

\section{Applications}

Les méthodes utilisées dans le présent travail sont aisément généralisables aux cas où la série de Dirichlet en cause peut être exprimée comme le carré d'une série à coefficients positifs ou nuls. Décrivons ici deux applications liées à des fonctions arithmétiques classiques.

Considérons d'abord la fonction nombre de diviseurs, $n \mapsto \tau(n)$. Nous pouvons estimer, dans les mêmes domaines que précédemment, la fonction sommatoire friable

$$
\Psi_{\tau}(x, y):=\sum_{n \in S(x, y)} \tau(n) .
$$

Le terme principal attendu est $Q_{\tau, y}(\log x)$, un polynôme en $\log x$ de degré $2 h=2 \pi(y)$. Parallèlement à $(2 \cdot 6)$, nous obtenons

$$
Q_{\tau, y}(\xi)=\sum_{0 \leqslant k \leqslant 2 h} \frac{\xi^{2 h-k} d_{k}(y)}{(2 h-k) ! \prod_{p \leqslant y}(\log p)^{2}},
$$

où l'on a posé, avec la notation $(2 \cdot 7)$,

$$
d_{k}(y):=\sum_{0 \leqslant j \leqslant k} c_{j}(y) c_{k-j}(y) \quad(k \geqslant 0) .
$$


L'analogue du Théorème 2.1 s'énonce alors comme suit.

Théorème 4.1. Pour chaque $\varepsilon>0$ et sous la condition (1·1), nous avons

$$
\Psi_{\tau}(x, y)=\left\{1+O\left(\frac{1}{Y_{\varepsilon} \log x}\right)\right\} Q_{\tau, y}(\log x) .
$$

En particulier, notant $h:=\pi(y)$, nous avons

$$
Q_{\tau, y}(\xi)=\frac{\left\{\xi+\vartheta_{1}(y)\right\}^{2 h}}{(2 h) ! \prod_{p \leqslant y}(\log p)^{2}}\left\{1+O\left(\frac{y^{2} h}{\xi^{2}}\right)\right\} \quad(\xi \geqslant 1) .
$$

Remarque. Une évaluation analogue peut être obtenue pour la sous-somme restreinte aux entiers $y$-friables premiers à un entier $m$ fixé.

Il résulte en particulier des Théorèmes 2.1 et 4.1 que, lorsque $y=o\left(\sqrt{\log x \log _{2} x}\right)$, nous avons

$$
\frac{\Psi_{\tau}(x, y)}{\Psi(x, y)}=\frac{h !(\log x)^{h}}{(2 h) ! \prod_{p \leqslant y} \log p}\left\{1+O\left(\frac{h y}{\log x}\right)\right\}=\left(\frac{\log x}{y}\right)^{h+o(h)} \quad(x \rightarrow \infty) .
$$

Cela précise, dans le cas particulier et pour le domaine considérés, l'évaluation du théorème 2.5 de [1], soit

$$
\frac{\Psi_{\tau}(x, y)}{\Psi(x, y)}=\zeta(\alpha, y)\left\{1+O\left(\frac{1}{h}\right)\right\}
$$

dont le terme résiduel ne tend pas vers 0 lorsque $y$ est borné. Qualitativement, ces résultats fournissent une nouvelle illustration du fait qu'un entier très friable possède en moyenne un nombre anormalement grand de diviseurs.

Notre seconde application concerne l'estimation de

$$
\Psi_{r}(x, y):=\sum_{n \in S(x, y)} r(n)
$$

où $r(n)$ désigne le nombre de représentations de l'entier $n$ comme somme de deux carrés. Notons, pour $y \geqslant 2$,

$$
\begin{aligned}
& \pi(y ; j, 4):=\sum_{\substack{p \leqslant y \\
p \equiv j(\bmod 4)}} 1 \quad(j=3,4), \quad \nu_{p}:= \begin{cases}2 & \text { si } p \equiv 1(\bmod 4) \\
1 & \text { si } p=2 \text { ou } p \equiv 3(\bmod 4),\end{cases} \\
& h_{4}:=\sum_{p \leqslant y} \nu_{p}=\pi(y)+\pi(y ; 1,4), \\
& b_{k}(y):=(-1)^{k} \sum_{\dagger k} \prod_{p \leqslant y} \frac{B_{j_{p}} w_{p}\left(j_{p}\right)(-1)^{m_{p}}(\log p)^{j_{p}+m_{p}}}{j_{p} ! m_{p} !}(k \geqslant 0),
\end{aligned}
$$

où le symbole $\dagger k$ signifie que la sommation porte sur toutes les suites de vecteurs $\left\{\left(j_{p}, m_{p}\right)\right\}_{p \leqslant y} \in \mathbb{N}^{\pi(y)} \times \mathbb{N}^{\pi(y ; 1,4)}$ telles que $\sum_{p \leqslant y} j_{p}+\sum_{p \leqslant y, p \equiv 1(\bmod 4)} m_{p}=k$ et où l'on a posé

$$
w_{p}(j):= \begin{cases}1 & \text { si } p=2 \\ 1-j & \text { si } p \equiv 1(\bmod 4) \\ 2^{j} & \text { si } p \equiv 3(\bmod 4) .\end{cases}
$$


Le terme principal attendu dans l'évaluation de (4.6) fait intervenir le polynôme de degré $h_{4}$ défini par

$$
Q_{r, y}(\xi):=\frac{2^{2-\pi(y ; 3,4)}}{\prod_{p \leqslant y}(\log p)^{\nu_{p}}} \sum_{0 \leqslant k \leqslant h_{4}} \frac{\xi^{h_{4}-k} b_{k}(y)}{\left(h_{4}-k\right) !}
$$

Théorème 4.2. Pour chaque $\varepsilon>0$ et sous la condition (1·1), nous avons

$$
\Psi_{r}(x, y)=\left\{1+O\left(\frac{1}{Y_{\varepsilon} \log x}\right)\right\} Q_{r, y}(\log x) .
$$

De plus,

$$
Q_{r, y}(\xi)=\frac{4\left\{\xi+\vartheta_{1}(y)-\frac{1}{2} \log 2\right\}^{h_{4}}}{h_{4} ! 2^{\pi(y ; 3,4)} \prod_{p \leqslant y}(\log p)^{\nu_{p}}}\left\{1+O\left(\frac{y^{2} h_{4}}{\xi^{2}}\right)\right\} \quad(\xi \geqslant 1) .
$$

Il résulte en particulier des Théorèmes 2.1 et 4.2 que, lorsque $y=o\left(\sqrt{\log x \log _{2} x}\right)$, nous avons

$$
\begin{aligned}
\frac{\Psi_{r}(x, y)}{\Psi(x, y)} & =\frac{4(\log x)^{\pi(y ; 1,4)} h !}{h_{4} ! 2^{\pi(y ; 3,4)} \prod_{\substack{p \leqslant y \\
p \equiv 1 \bmod 4}} \log p}\left\{1+O\left(\frac{h_{4} y}{\log x}\right)\right\} \\
& =\left(\frac{\log x}{y}\right)^{\{1+o(1)\} \pi(y ; 1,4)} \quad(x \rightarrow \infty) .
\end{aligned}
$$

Par comparaison avec $(4 \cdot 5)$, nous obtenons ainsi que l'ordre moyen friable de $r(n)$ est comparable à la racine carrée de celui de $\tau(n)$ pour les petites valeurs du paramètre $y$.

\section{Preuves}

\section{5·1. Démonstration du Théorème 2.1}

Pour des raisons techniques, nous faisons appel à la variante de la formule $(2 \cdot 1)$

$$
\int_{0}^{x} \Psi(t, y) \mathrm{d} t=\frac{1}{2 \pi i} \int_{\alpha-i \infty}^{\alpha+i \infty} \frac{\zeta(s, y) x^{s+1}}{s(s+1)} \mathrm{d} s
$$

qui est valable pour tout $x>0$. L'étape liminaire de la preuve consiste à tronquer l'intégrale du membre de droite de $(5 \cdot 1)$. À cette fin, nous observons d'abord qu'il existe une constante absolue $y_{0}$ telle que, si $y>y_{0}$, nous ayons pour tout $\varepsilon>0$ et une constante convenable $c=c_{\varepsilon}>0$,

$$
\frac{\zeta(s, y)}{\zeta(\alpha, y)} \ll\left(1+\frac{\tau^{2}(\log x)^{2}}{\left(1+\tau^{2}\right) y^{2}}\right)^{-c \pi(y)} \quad\left(2 \leqslant y \leqslant \log x, \sigma=\alpha,|\tau| \leqslant Y_{\varepsilon}^{2}\right) .
$$

En effet, compte tenu de l'évaluation connue $\alpha \asymp \pi(y) / \log x$, valable dans le domaine $2 \leqslant y \leqslant \log x-\operatorname{voir}(2 \cdot 2)$ - et de l'identité

$$
\left(\frac{1-p^{-\alpha}}{\left|1-p^{-s}\right|}\right)^{2}=\frac{1}{1+4\left(\sin \frac{1}{2} \tau \log p\right)^{2} / p^{\alpha}\left(1-p^{-\alpha}\right)^{2}} \quad(p \in \mathbb{P}, s=\alpha+i \tau)
$$

la majoration $(5 \cdot 2)$ est évidente sous la condition supplémentaire $|\tau| \leqslant \pi / \log y$. Lorsque $\pi / \log y<|\tau| \leqslant \sqrt{y}$ et $y_{0}$ est assez grand, nous pouvons employer les majorations classiques pour le nombre de nombres premiers dans les petits intervalles. Nous omettons les détails, analogues à ceux de la preuve du lemme 5.12 de [18]. Enfin, dans le domaine 
$\sqrt{y} \leqslant|\tau| \leqslant Y_{\varepsilon}^{2}$, nous utilisons d'une part l'estimation, suivante, valable pour une constante $c_{0}>0$ convenable et établie par la méthode usuelle d'intégration complexe à partir de la région sans zéro de Vinogradov-Korobov,

$$
\sum_{p \leqslant y} p^{i \tau} \ll y^{1-c_{0} \beta(\tau)}+\frac{\pi(y)}{3+|\tau|}+y^{1-c_{0} \beta(y)} \quad\left(y \geqslant 3, \tau \in \mathbb{R}, \log (3+|\tau|) \leqslant \frac{(\log y)^{3 / 2}}{\left(\log _{2} y\right)^{2}}\right),
$$

où l'on a posé $\beta(\tau):=\{\log (3+|\tau|)\}^{-2 / 3}\left\{\log _{2}(3+|\tau|)\right\}^{-1 / 3}$, et, d'autre part, l'inégalité d'Erdős-Turán - voir, par exemple, [19], th. I.6.15. Nous obtenons ainsi, dans le domaine $\sqrt{y} \leqslant|\tau| \leqslant \mathrm{e}^{(\log y)^{3 / 2} /\left(\log _{2} y\right)^{3}}$,

$$
\sum_{\substack{p \leqslant y \\\left\|(\tau / 2 \pi) \log p-\frac{1}{4}\right\| \leqslant t}} 1=t \pi(y)+O\left(\frac{\pi(y)}{(\log y)^{2}}\right) \quad\left(y \geqslant 2,0 \leqslant t \leqslant \frac{1}{2}\right) .
$$

Cela suffit pleinement à établir $(5 \cdot 2)$ en choisissant par exemple $t=\frac{1}{6}$ puis $y_{0}$ assez grand. Nous notons incidemment que la majoration $(5 \cdot 2)$ est valable dans le domaine étendu $y \leqslant \log x$, mais nous n'utiliserons pas cela dans la suite.

Il découle de $(5 \cdot 2)$ que

$$
\int_{-Y_{\varepsilon}^{2}}^{Y_{\varepsilon}^{2}}\left|x^{s} \zeta(s, y)\right| \mathrm{d} \tau \ll \frac{x^{\alpha} \zeta(\alpha, y) y}{\sqrt{\pi(y)} \log x} \ll \frac{\Psi(x, y) y}{\log x} \ll \frac{\Psi(x, y) Y_{\varepsilon}}{\log x},
$$

où la seconde majoration est issue de l'évaluation

$$
\Psi(x, y) \asymp \frac{x^{\alpha} \zeta(\alpha, y) \sqrt{\pi(y)}}{\alpha \log x} \asymp \frac{x^{\alpha} \zeta(\alpha, y)}{\sqrt{\pi(y)}},
$$

établie dans [13] sous l'hypothèse $2 \leqslant y \leqslant \log x$.

Introduisons à présent la fonction arithmétique multiplicative $\mathfrak{r}$ définie par

$$
\mathfrak{r}\left(p^{\nu}\right)=\mathfrak{r}_{\nu}:=\left(\begin{array}{c}
2 \nu \\
\nu
\end{array}\right) / 4^{\nu} \quad(\nu \geqslant 0, p \in \mathbb{P}) .
$$

Nous avons

$$
Z(s, y):=\sum_{n \geqslant 1} \frac{\mathfrak{r}(n)}{n^{s}}=\zeta(s, y)^{1 / 2} \quad(\sigma>0, y \geqslant 2)
$$

et, puisque $\mathfrak{r} \geqslant 0$, nous pouvons écrire en vertu de l'inégalité de Montgomery-Wirsing (voir [16], th. 7.3 ou [19], lemme III.4.10),

$$
\begin{aligned}
\int_{U}^{U+2 V}|\zeta(s, y)| \mathrm{d} \tau & =\int_{U}^{U+2 V}|Z(s, y)|^{2} \mathrm{~d} \tau \\
& \leqslant 3 \int_{-V}^{V}|Z(s, y)|^{2} \mathrm{~d} \tau=3 \int_{-V}^{V}|\zeta(s, y)| \mathrm{d} \tau
\end{aligned} \quad(U \in \mathbb{R}, V>0) .
$$

Compte tenu de $(5 \cdot 3)$, nous en déduisons que

$$
\int_{\alpha+i k Y_{\varepsilon}^{2}}^{\alpha+i(k+1) Y_{\varepsilon}^{2}} \frac{\zeta(s, y) x^{s+1}}{s(s+1)} \mathrm{d} s \ll \frac{x \Psi(x, y)}{k^{2} Y_{\varepsilon}^{2} \log x} \quad(k \in \mathbb{Z}, k \neq-1,0) .
$$


D'autre part, grâce à $(5 \cdot 2)$, nous avons

$$
\int_{\alpha \pm i / \log y}^{\alpha \pm i Y_{\varepsilon}^{2}} \frac{\zeta(s, y) x^{s+1}}{s(s+1)} \mathrm{d} s \ll x^{1+\alpha} \zeta(\alpha, y)\left(\log _{2} 2 y\right)\left(\frac{\log x}{2 y \log y}\right)^{-2 c \pi(y)} \ll \frac{x \Psi(x, y)}{Y_{\varepsilon}^{2} \log x}
$$

En reportant dans $(5 \cdot 1)$ et en tenant compte de $(5 \cdot 2)$, nous obtenons, dans le domaine $y_{0}<y \leqslant(\log x)^{1-\varepsilon}$,

$$
\int_{0}^{x} \Psi(t, y) \mathrm{d} t=\frac{1}{2 \pi i} \int_{\alpha-i / \log y}^{\alpha+i / \log y} \frac{\zeta(s, y) x^{s+1}}{s(s+1)} \mathrm{d} s+O\left(\frac{x \Psi(x, y)}{Y_{\varepsilon}^{2} \log x}\right) .
$$

Pour montrer que $(5 \cdot 4)$ persiste pour $2 \leqslant y \leqslant y_{0}$, il suffit de remplacer, dans les calculs qui précèdent, $Y_{\varepsilon}^{2}$ par $\pi / \log y_{0}$, de sorte que $(5 \cdot 2)$ est trivialement valable. Comme cette manipulation n'altère que la constante implicite dans $(5 \cdot 4)$, la conclusion est acquise.

Déplaçons alors le segment d'intégration vers la gauche jusqu'à l'abscisse $\sigma=-1 / \log y$. Le seul résidu à prendre en compte est en $s=0$. La contribution des segments horizontaux peut être évaluée en observant que

$$
\left|\zeta\left(\sigma \pm \frac{i}{\log y}, y\right)\right| \leqslant \prod_{p \leqslant y} \frac{p^{\sigma / 2}}{2 \sin \{(\log p) /(2 \log y)\}} \ll \mathrm{e}^{c_{1} \pi(y)} \quad(-1 / \log y \leqslant \sigma \leqslant \alpha)
$$

pour tout $c_{1}>\log (\pi / 2)$. Celle du segment vertical relève de la majoration

$$
|\zeta(s, y)| \leqslant \prod_{p \leqslant y} \frac{1}{p^{1 / \log y}-1} \ll \mathrm{e}^{c_{1} \pi(y)} \quad(s=-1 / \log y+i \tau) .
$$

Comme $\zeta(\alpha, y) \gg(\log x)^{\varepsilon \pi(y) / 2}+(\log x)^{\pi(y)} \mathrm{e}^{-y+O(y / \log y)}$ dans le domaine (1·1), nous obtenons ainsi

$$
\int_{0}^{x} \Psi(t, y) \mathrm{d} t=\int_{0}^{x} P_{y}(\log t) \mathrm{d} t+O\left(\frac{x \Psi(x, y)}{Y_{\varepsilon / 2}^{2} \log x}\right) .
$$

En utilisant l'encadrement

$$
\frac{1}{z} \int_{x-z}^{x} \Psi(t, y) \mathrm{d} t \leqslant \Psi(x, y) \leqslant \frac{1}{z} \int_{x}^{x+z} \Psi(t, y) \mathrm{d} t
$$

avec $z:=x / Y_{2 \varepsilon / 3}$ et l'estimation

$$
P_{y}^{\prime}(\log x) \ll \frac{\pi(y)}{\log x} P_{y}(\log x),
$$

valable dans le domaine $(1 \cdot 1)$, nous obtenons bien $(2 \cdot 9)$.

Pour établir (5.5), nous écrivons

$$
P_{y}(\log x)=\frac{1}{2 \pi i} \int_{R} \frac{x^{s} \zeta(s, y)}{s} \mathrm{~d} s, \quad P_{y}^{\prime}(\log x)=\frac{1}{2 \pi i} \int_{R} x^{s} \zeta(s, y) \mathrm{d} s,
$$

où $R$ est le rectangle de sommets $\alpha \pm i / \log y,(-1 \pm i) / \log y$, parcouru dans le sens direct.

Considérons d'abord la première intégrale de (5·6). La contribution globale des segments horizontaux et du segment vertical gauche est majorée comme précédemment par

$$
\ll x^{\alpha} \mathrm{e}^{c_{2} \pi(y)} \ll \frac{x^{\alpha} \zeta(\alpha, y)}{\sqrt{\pi(y)}(\log x)^{\varepsilon \pi(y) / 2}},
$$


pour toute constante $c_{2}>1$. D'après le lemme 10 de [13], la contribution du segment vertical droit est de l'ordre de

$$
\frac{x^{\alpha} \zeta(\alpha, y)}{\sqrt{\pi(y)}} \asymp \Psi(x, y)
$$

Nous pouvons donc écrire, dans le domaine $(1 \cdot 1)$,

$$
P_{y}(\log x) \asymp \Psi(x, y) .
$$

La seconde intégrale de $(5 \cdot 6)$ peut être traitée similairement pour fournir

$$
P_{y}^{\prime}(\log x) \asymp \alpha \Psi(x, y) \asymp \frac{\pi(y) \Psi(x, y)}{\log x} .
$$

\subsection{Démonstration du Théorème 2.3}

L'estimation $(2 \cdot 16)$ est obtenue en appliquant à $\zeta_{m}(s, y)$ la méthode employée pour $\zeta(s, y)$ dans la démonstration du Théorème 2.1. Les calculs étant identiques, nous omettons les détails. Nous obtenons $(2 \cdot 16)$ avec le terme principal

$$
\begin{aligned}
P_{y, m}(\xi) & :=\operatorname{Rés}\left(\frac{\mathrm{e}^{\xi s}}{s} \zeta_{m}(s, y) ; 0\right)=\sum_{d \mid m} \mu(d) P_{y}(\xi-\log d) \\
& =\sum_{0 \leqslant k \leqslant \pi(y)-\omega(m)} \frac{\xi^{\pi(y)-\omega(m)-k} c_{k}(y ; m)}{(\pi(y)-\omega(m)-k) ! \prod_{\substack{p \leqslant y \\
p \nmid m}} \log p},
\end{aligned}
$$

où la seconde égalité est l'analogue de $(2 \cdot 6)$ pour $P_{y, m}$.

Nous obtenons $(2 \cdot 17)$ en observant que

$$
\operatorname{Rés}\left(\frac{\mathrm{e}^{\xi s}}{s} \zeta_{m}(s, y) ; 0\right)=\sum_{d \mid m} \mu(d) \operatorname{Rés}\left(\frac{\mathrm{e}^{(\xi-\log d) s}}{s} \zeta(s, y) ; 0\right) \text {. }
$$

\subsection{Démonstration du Théorème 2.4}

L'énoncé suivant explicite les premières valeurs des $c_{k}(y ; m)$.

Lemme 5.1. Soit $M \geqslant 1$. Sous les conditions $m \geqslant 1,0 \leqslant \omega(m) \leqslant M$ et $P^{+}(m) \leqslant y$, nous avons

$$
\begin{aligned}
& c_{0}(y ; m)=1, \\
& c_{1}(y ; m)=\frac{1}{2} \vartheta_{1}(y ; m), \\
& c_{2}(y ; m)=\frac{1}{8} \vartheta_{1}(y ; m)^{2}-\frac{1}{24} \vartheta_{2}(y ; m), \\
& c_{3}(y ; m)=\frac{1}{48} \vartheta_{1}(y ; m)\left\{\vartheta_{1}(y ; m)^{2}-\vartheta_{2}(y ; m)\right\} .
\end{aligned}
$$

Démonstration. Nous avons $B_{1}=-\frac{1}{2}, B_{2}=\frac{1}{6}, B_{3}=0$, et

$$
c_{0}(y ; m)=1, \quad c_{1}(y ; m)=-B_{1} \vartheta_{1}(y ; m)=\frac{1}{2} \vartheta_{1}(y ; m) .
$$

L'analyse des conditions $* 2$ permet d'écrire

$$
\begin{aligned}
c_{2}(y ; m) & =\frac{1}{2} B_{2} \vartheta_{2}(y ; m)+B_{1}^{2} \sum_{\substack{p_{1}<p_{2} \leqslant y \\
\left(p_{1} p_{2}, m\right)=1}}\left(\log p_{1}\right)\left(\log p_{2}\right) \\
& =\frac{1}{2} B_{2} \vartheta_{2}(y ; m)+\frac{1}{2} B_{1}^{2}\left\{\vartheta_{1}(y ; m)^{2}-\vartheta_{2}(y ; m)\right\}=\frac{1}{8} \vartheta_{1}(y ; m)^{2}-\frac{1}{24} \vartheta_{2}(y ; m) .
\end{aligned}
$$


Comme $B_{3}=0$, nous obtenons de même

$$
\begin{aligned}
c_{3}(y ; m)= & -\frac{1}{2} B_{1} B_{2}\left\{\vartheta_{1}(y ; m) \vartheta_{2}(y ; m)-\vartheta_{3}(y ; m)\right\} \\
& -B_{1}^{3} \sum_{\substack{p_{1}<p_{2}<p_{3} \leqslant y \\
\left(p_{1} p_{2} p_{3}, m\right)=1}}\left(\log p_{1}\right)\left(\log p_{2}\right)\left(\log p_{3}\right) \\
= & -\frac{1}{2} B_{1} B_{2}\left(\vartheta_{1}(y ; m) \vartheta_{2}(y ; m)-\vartheta_{3}(y ; m)\right) \\
& -\frac{1}{6} B_{1}^{3}\left(\vartheta_{1}(y ; m)^{3}-3 \vartheta_{1}(y ; m) \vartheta_{2}(y ; m)+2 \vartheta_{3}(y ; m)\right) \\
= & \frac{1}{48} \vartheta_{1}(y ; m)^{3}-\frac{1}{48} \vartheta_{1}(y ; m) \vartheta_{2}(y ; m) .
\end{aligned}
$$

Cela achève la démonstration du lemme.

Nous sommes maintenant en mesure de démontrer le Théorème 2.4.

Pour établir $(2 \cdot 22)$, nous pouvons manifestement supposer $y$ assez grand, et, compte tenu de $(5 \cdot 12), k \geqslant 2$. Nous observons alors que, d'après $(2 \cdot 4)$, la formule de Cauchy permet d'écrire, pour tout $r \in] 0,2 \pi / \log y[$,

$$
c_{k}(y ; m)=\frac{1}{2 \pi i} \oint_{|z|=r} f_{y, m}(z) \frac{\mathrm{d} z}{z^{k+1}}
$$

avec

$$
\begin{aligned}
f_{y, m}(z) & :=\prod_{\substack{p \leqslant y \\
p \nmid m}} \frac{z \log p}{1-p^{-z}}=\mathrm{e}^{z \vartheta_{1}(y ; m) / 2} \prod_{\substack{p \leqslant y \\
p \nmid m}} \frac{(z / 2) \log p}{\operatorname{sh}\{(z / 2) \log p\}} \\
& =\mathrm{e}^{z \vartheta_{1}(y ; m) / 2}\left\{1+O\left(z^{2} \vartheta_{2}(y ; m)\right)\right\} \quad(z \log y \ll 1) .
\end{aligned}
$$

La contribution du terme principal au membre de droite de $(5 \cdot 13)$ coïncide avec celui de $(2 \cdot 22)$. Pour évaluer le terme résiduel, nous choisissons $r:=2 k / \vartheta_{1}(y ; m)$. Nous obtenons la majoration

$$
\ll \frac{\mathrm{e}^{k} \vartheta_{1}(y ; m)^{k} k^{2}}{h(2 k)^{k}} \int_{-\pi}^{\pi} \mathrm{e}^{-(1-\cos v) k} \mathrm{~d} v \ll \frac{\vartheta_{1}(y ; m)^{k} k^{2}}{h 2^{k} k !}
$$

ce qui achève la preuve.

L'estimation $(2 \cdot 23)$ pour $P_{y, m}(\xi)$ en résulte par sommation sur $k$ grâce à la formule $d u$ binôme et à l'identité

$$
\sum_{0 \leqslant k \leqslant h} k(k-1)\left(\begin{array}{l}
h \\
k
\end{array}\right) a^{k} b^{h-k}=h(h-1) a^{2}(a+b)^{h-2} .
$$

Un développement à l'ordre 4 de l'intégrande de (5·13) permet d'affiner (2.23) pour obtenir $(2 \cdot 24)$.

\subsection{Démonstration du Corollaire 3.2}

Le lemme suivant fournit une approximation au premier ordre du rapport des termes principaux des approximations de $\Psi(x, y)$ et $\Psi_{m}(x, y)$ apparaissant respectivement en $(2 \cdot 9)$ et $(2 \cdot 16)$.

Lemme 5.2. Soit $\varepsilon>0$. Sous les conditions $\xi:=\log x>2,2 \leqslant y \leqslant \xi^{1-\varepsilon}, P^{+}(m) \leqslant y$, $\mu(m)^{2}=1, \omega(m) \ll 1$, nous avons uniformément

$$
P_{y, m}(\xi)=\frac{g_{m}(\alpha) P_{y}(\xi) h !}{\{h-\omega(m)\} ! h^{\omega(m)}}\left\{1+\frac{\omega(m) \log m}{2 \xi}+O\left(\frac{y^{2} h}{\xi^{2}}\right)\right\} .
$$


Démonstration. Observons d'abord que la définition de $\alpha=\alpha(x, y)$ et le développement $(2 \cdot 4)$ impliquent, pour $y / \xi$ assez petit, puisque $\alpha^{r} \vartheta_{r}(y) \ll h(2 y / \xi)^{r}(r \geqslant 0)$,

$$
\alpha \xi=\sum_{p \leqslant y} \frac{\alpha \log p}{p^{\alpha}-1}=\sum_{r \geqslant 0} \frac{B_{r}}{r !} \alpha^{r} \vartheta_{r}(y),
$$

de sorte qu'un développement à l'ordre 2 fournit

$$
\alpha \xi=\sum_{p \leqslant y} \frac{\alpha \log p}{p^{\alpha}-1}=h-\frac{1}{2} \alpha \vartheta_{1}(y)+O\left(\frac{y^{3}}{\xi^{2} \log y}\right),
$$

et donc

$$
\begin{aligned}
\alpha\left\{\xi+\frac{1}{2} \vartheta_{1}(y)\right\} & =h\left\{1+O\left(\frac{y^{2}}{\xi^{2}}\right)\right\} \quad(2 \leqslant y \leqslant \xi), \\
\alpha & =\frac{h}{\xi}\left\{1-\frac{\vartheta_{1}(y)}{2 \xi}+O\left(\frac{y^{2}}{\xi^{2}}\right)\right\} \quad(2 \leqslant y \leqslant \xi) .
\end{aligned}
$$

Il suit

$$
g_{p}(\alpha)=\frac{h \log p}{\xi+\frac{1}{2} \vartheta_{1}(y)}\left\{1-\frac{h \log p}{2 \xi}+O\left(\frac{y^{2}}{\xi^{2}}\right)\right\} \quad(p \leqslant y),
$$

et donc, dans les conditions de l'énoncé,

$$
g_{m}(\alpha)=h^{\omega(m)} \prod_{p \mid m} \frac{\log p}{\xi+\frac{1}{2} \vartheta_{1}(y)}\left\{1-\frac{h \log m}{2 \xi}+O\left(\frac{y^{2}}{\xi^{2}}\right)\right\} .
$$

Effectuons alors le rapport de l'évaluation $(2 \cdot 23)$ à sa spécialisation pour $m=1$. Nous obtenons, compte tenu de $(5 \cdot 19)$,

$$
\begin{aligned}
\frac{P_{y, m}(\xi)}{P_{y}(\xi)} & =\left(1-\frac{\log m}{2 \xi+\vartheta_{1}(y)}\right)^{h-\omega(m)} \frac{h !}{(h-\omega(m)) !} \prod_{p \mid m} \frac{\log p}{\xi+\frac{1}{2} \vartheta_{1}(y)}\left\{1+O\left(\frac{y^{2} h}{\xi^{2}}\right)\right\} \\
& =\frac{\{1-(h-\omega(m))(\log m) / 2 \xi\} h ! g_{m}(\alpha)}{\{1-h(\log m) / 2 \xi\}(h-\omega(m)) ! h^{\omega(m)}}\left\{1+O\left(\frac{y^{2} h}{\xi^{2}}\right)\right\} \\
& =\left(1+\frac{\omega(m) \log m}{2 \xi}\right) \frac{h ! g_{m}(\alpha)}{(h-\omega(m)) ! h^{\omega(m)}}\left\{1+O\left(\frac{y^{2} h}{\xi^{2}}\right)\right\} .
\end{aligned}
$$

Cela implique bien $(5 \cdot 14)$.

Nous sommes à présent en mesure de prouver le Corollaire 3.2. Nous pouvons nous restreindre au cas $y \leqslant \sqrt{\log x \log _{2} x}$ : la circonstance complémentaire relève du théorème 2.4 de [1] puisque l'on a alors $\mathfrak{R} \asymp 1 / u_{y}+t / u$.

L'estimation $(2 \cdot 23)$ fournit alors, sous les hypothèses considérées, notant $\xi=\log x$,

$$
\begin{aligned}
\frac{P_{y, m}(\xi-t \log y)}{P_{y, m}(\xi)} & =\left(1-\frac{2 \log d}{2 \xi+\vartheta_{1}(y)-\log m}\right)^{h-\omega(m)}\left\{1+O\left(\frac{y^{2} h}{\xi^{2}}\right)\right\} \\
& =\frac{1}{d^{\beta}}\left(1-\frac{t^{2}}{u^{2}+\bar{u}^{2}}\right)^{b \bar{u}}\left\{1+O\left(\frac{y^{2} h}{\xi^{2}}\right)\right\},
\end{aligned}
$$

avec $\beta:=2\{h-\omega(m)\} /\left(2 \xi+\vartheta_{1}(y)-\log m\right)$. Comme (5·16) implique

nous avons

$$
d^{h /\left(2 \xi+\vartheta_{1}(y)\right)}=d^{\alpha}\left\{1+O\left(\frac{y^{2} h}{\xi^{2}}\right)\right\}
$$

$$
d^{\beta}=d^{\alpha}\left\{1+O\left(\frac{t \omega(m)}{u}+\frac{y^{2} h}{\xi^{2}}\right)\right\} .
$$

La conclusion requise résulte alors du Théorème 2.3 et du Lemme 5.2. 


\subsection{Comparaison avec la méthode du col : preuve de la Proposition 2.2}

Considérons un couple $(x, y)$ vérifiant $(1 \cdot 1)$ et, pour simplifier les écritures, posons encore

$$
h:=\pi(y) .
$$

Constatons d'abord que nous pouvons restreindre l'étude au domaine (2·11). En effet, lorsque $y \gg \sqrt{\log x \log _{2} x}$, les relations $(2 \cdot 15)$ résultent de $(2 \cdot 13)$ et du Théorème 2.1 puisque $\mathfrak{s}(h)=1+O(1 / h)$ et $y^{2} h /(\log x)^{2} \gg 1 / h$. De plus, nous pouvons clairement supposer $x$ assez grand.

Comme le développement de $1 /\left(\mathrm{e}^{s}-1\right)$ fournit, par dérivation,

$$
\frac{s^{2} \mathrm{e}^{s}}{\left(\mathrm{e}^{s}-1\right)^{2}}=\sum_{r \geqslant 0} \frac{B_{r}}{r !}(1-r) s^{r},
$$

nous avons

$$
\alpha^{2} \sigma_{2}=\sum_{p \leqslant y} \frac{(\alpha \log p)^{2} p^{\alpha}}{\left(p^{\alpha}-1\right)^{2}}=h+\sum_{r \geqslant 2} \frac{B_{r}}{r !}(1-r) \alpha^{r} \vartheta_{r}(y) .
$$

Compte tenu de la majoration classique $B_{r} / r$ ! $\ll 1 /(2 \pi)^{r}(r \geqslant 1)$ et de l'estimation (5.17), la dernière somme en $r$ est $\ll h(y / \log x)^{2}$. Elle n'excède donc pas $\frac{1}{2} h$ en valeur absolue lorsque $(x, y)$ vérifie $(2 \cdot 11)$ et $x$ est assez grand. Le développement

$$
\frac{1}{\sqrt{1-z}}=\sum_{\nu \geqslant 0}\left(\begin{array}{c}
2 \nu \\
\nu
\end{array}\right) \frac{z^{\nu}}{4^{\nu}} \quad(|z|<1)
$$

permet alors d'écrire

$$
\begin{aligned}
\frac{1}{\sqrt{2 \pi \alpha^{2} \sigma_{2}}} & =\frac{1}{\sqrt{2 \pi h}} \sum_{\nu \geqslant 0}\left(\begin{array}{c}
2 \nu \\
\nu
\end{array}\right) \frac{1}{4^{\nu}}\left(\sum_{r \geqslant 2} \frac{B_{r}}{r !}(r-1) \alpha^{r} \frac{\vartheta_{r}(y)}{h}\right)^{\nu} \\
& =\frac{1}{\sqrt{2 \pi h}}\left\{1+O\left(\frac{y^{2}}{(\log x)^{2}}\right)\right\} .
\end{aligned}
$$

Maintenant, la formule $(2 \cdot 5)$ appliquée en $s=\alpha$ implique, avec la notation $(2 \cdot 7)$,

$$
\zeta(\alpha, y) \prod_{p \leqslant y} \log p=\frac{1}{\alpha^{h}} \sum_{k \geqslant 0}(-1)^{k} \alpha^{k} \sum_{* k} \prod_{p \leqslant y} \frac{B_{j_{p}}(\log p)^{j_{p}}}{j_{p} !}=\frac{1}{\alpha^{h}} \sum_{k \geqslant 0} \alpha^{k} c_{k}(y) .
$$

En reportant l'estimation $(2 \cdot 22)$, nous obtenons

$$
\begin{aligned}
\zeta(\alpha, y) \prod_{p \leqslant y} \log p & =\frac{1}{\alpha^{h}} \sum_{k \geqslant 0} \alpha^{k} \frac{\vartheta_{1}(y)^{k}}{2^{k} k !}\left\{1+O\left(\frac{k(k-1) \log y}{y}\right)\right\} \\
& =\frac{\mathrm{e}^{\alpha \vartheta_{1}(y) / 2}}{\alpha^{h}}\left\{1+O\left(\frac{y^{2} h}{(\log x)^{2}}\right)\right\} .
\end{aligned}
$$

Au vu de (5·16), nous avons, dans le domaine $(2 \cdot 11)$,

$$
x^{\alpha} \mathrm{e}^{\alpha \vartheta_{1}(y) / 2}=\exp \left\{h+O\left(\frac{y^{2} h}{(\log x)^{2}}\right)\right\}=\mathrm{e}^{h}\left\{1+O\left(\frac{y^{2} h}{(\log x)^{2}}\right)\right\},
$$

et aussi

$$
\left(\alpha\left\{\log x+\frac{1}{2} \vartheta_{1}(y)\right\}\right)^{h} h^{-h}=\left\{1+O\left(\frac{y^{2}}{(\log x)^{2}}\right)\right\}^{h}=1+O\left(\frac{y^{2} h}{(\log x)^{2}}\right) .
$$


Posant $\eta:=y^{2} h /(\log x)^{2}$, nous déduisons de $(2 \cdot 12),(5 \cdot 20),(5 \cdot 21)$ et $(5 \cdot 22)$ que

$$
M(x, y)=\frac{\mathrm{e}^{h}\{1+O(\eta)\}}{\alpha^{h} \sqrt{2 \pi h} \prod_{p \leqslant y} \log p},
$$

alors que, compte tenu de (5·23), l'évaluation $(2 \cdot 23)$ avec $m=1$ implique

$$
P_{y}(\log x)=\frac{h^{h}\{1+O(\eta)\}}{\alpha^{h} h ! \prod_{p \leqslant y} \log p}=\frac{\mathrm{e}^{h}\{1+O(\eta)\}}{\alpha^{h} \mathfrak{s}(h) \sqrt{2 \pi h} \prod_{p \leqslant y} \log p} .
$$

Nous avons donc bien établi $(2 \cdot 15)$.

\subsection{Démonstration du Théorème 4.1}

La quantité $Q_{\tau, y}(\log x)$ est le résidu en $s=0$ de la fonction $\zeta(s, y)^{2} x^{s} / s$. En effet, notant toujours $h:=\pi(y)$, nous avons

$$
\zeta(s, y) s^{h}=\sum_{j \geqslant 0} \frac{c_{j}(y) s^{j}}{\prod_{p \leqslant y} \log p}, \quad \zeta(s, y)^{2} s^{2 h}=\sum_{k \geqslant 0} \frac{d_{k}(y) s^{k}}{\prod_{p \leqslant y}(\log p)^{2}}
$$

où les coefficients $d_{k}(y)$ sont définis par $(4 \cdot 2)$. Nous n'indiquons pas plus de détails pour la preuve de $(4 \cdot 3)$.

Pour établir (4.4), nous déduisons de l'estimation (2.22) que, pour $0 \leqslant k \leqslant h$, nous avons

$$
\begin{aligned}
d_{k}(y) & =\frac{\vartheta_{1}(y)^{k}}{2^{k} k !} \sum_{0 \leqslant j \leqslant k}\left(\begin{array}{c}
k \\
j
\end{array}\right)\left\{1+O\left(\frac{(k-j)(k-j-1)}{h}\right)\right\}\left\{1+O\left(\frac{j(j-1)}{h}\right)\right\} \\
& =\frac{\vartheta_{1}(y)^{k}}{k !}\left\{1+O\left(\frac{k(k-1)}{h}+\frac{k(k-1)(k-2)(k-3)}{h^{2}}\right)\right\} .
\end{aligned}
$$

De plus, $d_{2}(y)=\frac{1}{2} \vartheta_{1}(y)^{2}-\frac{1}{12} \vartheta_{2}(y)$. Nous en déduisons bien (4·4) par sommation sur $k$.

\subsection{Démonstration du Théorème 4.2}

La preuve étant analogue à celle du Théorème 4.1, nous nous limitons à de succinctes indications. Soit $\chi_{4}$ le caractère de Dirichlet non principal de module 4 . La relation de convolution $r=4\left(1 * \chi_{4}\right)$ implique

$$
F_{r}(s ; y):=4 \sum_{P^{+}(n) \leqslant y} \frac{r(n)}{n^{s}}=4 \zeta(s ; y) L\left(s ; \chi_{4}, y\right),
$$

où l'on a posé $L\left(s ; \chi_{4}, y\right):=\sum_{P^{+}(n) \leqslant y} \chi_{4}(n) / n^{s}$. Nous utiliserons la représentation

$$
F_{r}(s ; y)=\frac{4}{1-2^{-s}} \prod_{\substack{p \leqslant y \\ p \equiv 1 \bmod 4}} \frac{1}{\left(1-p^{-s}\right)^{2}} \prod_{\substack{p \leqslant y \\ p \equiv 3 \bmod 4}} \frac{1}{1-p^{-2 s}} .
$$

Nous ferons usage du résultat suivant, qui est l'analogue pour la série $F_{r}(s, y)$ du résultat de Hildebrand et Tenenbaum [13] relatif à la série $\zeta(s, y)$. Nous désignons par $\alpha_{r}=\alpha_{r}(x, y)$ le point-selle associé à la fonction $F_{r}(s, y) x^{s}$, i.e. l'unique solution positive de l'équation

$$
\frac{\log 2}{2^{\alpha_{r}}-1}+\sum_{\substack{p \leqslant y \\ p \equiv 1(\bmod 4)}} \frac{2 \log p}{p^{\alpha_{r}}-1}+\sum_{\substack{p \leqslant y \\ p \equiv 3(\bmod 4)}} \frac{2 \log p}{p^{2 \alpha_{r}}-1}=\log x,
$$


rappelons la notation $h_{4}$ de $(4 \cdot 7)$, et notons

$$
\begin{aligned}
& u:=(\log x) / \log y, \quad \bar{u}:=\min (u, y / \log y), \quad v:=y / \log x \quad(x \geqslant y \geqslant 2), \\
& \varphi_{0, r}(s, y):=\log F_{r}(s, y), \quad \varphi_{k, r}(s, y):=\varphi_{0, r}^{(k)}(s, y), \quad(k \geqslant 0, \Re e s>0), \\
& \sigma_{k, r}:=\varphi_{k, r}\left(\alpha_{r}, y\right),
\end{aligned}
$$

où la branche du logarithme complexe est obtenue par addition des déterminations principales $\log \left\{1 /\left(1-p^{-j s}\right)\right\} \quad(j=1,2)$ pour $p \leqslant y$. Observons immédiatement qu'une approche parallèle à celles des lemmes 2 et 4 de [13] fournit l'ordre de grandeur

$$
\sigma_{k, r} \asymp(u \log y)^{k} /(\bar{u})^{k-1} \quad(k \geqslant 1) .
$$

Théorème 5.3. Uniformément pour $x \geqslant y \geqslant 2$, nous avons

$$
\Psi_{r}(x, y)=\frac{x^{\alpha_{r}} F\left(\alpha_{r}, y\right)}{\alpha_{r} \sqrt{2 \pi \sigma_{2, r}}}\left\{1+\left(\frac{1}{\bar{u}}\right)\right\} .
$$

De plus, pour tout $\varepsilon>0$, nous avons

$$
(5 \cdot 28) \alpha_{r}= \begin{cases}1-\frac{\xi(u)}{\log y}+O\left(\frac{1}{L_{\varepsilon}(y)}+\frac{1}{u(\log y)^{2}}\right) & \text { si }(\log x)^{1+\varepsilon} \leqslant y \leqslant x . \\ \frac{1}{\log y} \log \left(\sqrt{1+2 v+\frac{1}{4} v^{2}}+\frac{1}{2} v\right)\left\{1+O\left(\frac{1}{\log y}\right)\right\} & \text { si } 2 \leqslant y \leqslant(\log x)^{2}, \\ \frac{h_{4}}{\log x}\left\{1+O\left(\frac{y}{\log x}\right)\right\} & \text { si } 2 \leqslant y \leqslant \log x .\end{cases}
$$

Admettons momentanément ce résultat. Nous pouvons alors suivre, mutatis mutandis, la preuve du Théorème 2.1 pour obtenir (4.9) avec

$$
Q_{r, y}(\xi):=\operatorname{Rés}\left(\frac{\mathrm{e}^{\xi s}}{s} F_{r}(s ; y) ; 0\right) .
$$

Compte tenu du développement de Laurent à l'origine

$$
F_{r}(s ; y)=\frac{2^{2-\pi(y ; 3,4)}}{s^{h_{4}} \prod_{p \leqslant y}(\log p)^{\nu_{p}}} \sum_{k \geqslant 0} b_{k}(y) s^{k},
$$

nous en déduisons bien l'expression (4.8). Un calcul analogue à celui qui conduit à l'estimation $(2 \cdot 22)$ — voir le paragraphe 5.3 - fournit ensuite, pour $0 \leqslant k \leqslant h_{4}$,

$$
b_{k}(y)=\frac{\left\{\vartheta_{1}(y)-\frac{1}{2} \log 2\right\}^{k}}{k !}\left\{1+O\left(\frac{k(k-1)}{h_{4}}+\frac{k(k-1)(k-2)(k-3)}{h_{4}^{2}}\right)\right\} .
$$

Cela implique l'évaluation (4·10) annoncée par sommation sur $k$ dans (4·8).

Démonstration du Théorème 5.3.

La preuve étant calquée sur celle de [13], nous nous contentons d'indications succinctes.

Commençons par $(5 \cdot 28)$.

Pour la première estimation, les évaluation de [13] sont encore valables, aussi nous renvoyons à ce travail pour les détails. 
Dans le domaine $2 \leqslant y \leqslant(\log x)^{2}$, une intégration par parties et le théorème des nombres premiers en progression arithmétique fournissent l'estimation

$$
\frac{y^{1-\alpha_{r}}}{1-y^{-\alpha_{r}}}\left\{1+O\left(\frac{1}{\log y}\right)\right\}+\frac{y^{1-2 \alpha_{r}}}{1-y^{-2 \alpha_{r}}}\left\{1+O\left(\frac{1}{\log y}\right)\right\}=\log x
$$

d'où nous déduisons que $z=y^{\alpha_{r}}$ est solution de l'équation

$$
\frac{1}{z-1}+\frac{1}{z^{2}-1}=\frac{1}{w}
$$

où $w=\{1+O(1 / \log y)\} v$. Il suit

$$
z=\sqrt{1+2 w+\frac{1}{4} w^{2}}+\frac{1}{2} w
$$

ce qui implique l'estimation annoncée.

Enfin, compte tenu de la seconde sous la forme $\alpha_{r} \asymp \pi(y) / \log x$, la troisième estimation de $\alpha_{r}$ résulte immédiatement de $(5 \cdot 25)$.

Pour établir $(5 \cdot 27)$, nous devons majorer le rapport $\left|F_{r}(s, y)\right| / F_{r}\left(\alpha_{r} ; y\right)$ dans un intervalle aussi grand que possible en $\tau=\Im m s$. L'approche de [13] fonctionne sans changement majeur. Nous obtenons ainsi, pour chaque $\varepsilon>0$ fixé et avec la notation $(2 \cdot 8)$, la validité de la majoration

$$
\frac{F_{r}\left(\alpha_{r}+i \tau, y\right)}{F_{r}\left(\alpha_{r}, y\right)} \ll \begin{cases}\exp \left\{\frac{-c_{0} y}{\log y} \log \left(1+\frac{\tau^{2} \sigma_{2, r}}{y / \log y}\right)\right\} & (|\tau| \leqslant 1 / \log y) \\ \exp \left\{\frac{-c_{0} \tau^{2} \bar{u}}{(1-\alpha)^{2}+\tau^{2}}\right\} & \left(1 / \log y<|\tau| \leqslant Y_{\varepsilon}\right)\end{cases}
$$

uniformément lorsque $x \geqslant y \geqslant 2$.

La formule de Perron fournit alors l'estimation

$$
\Psi_{r}(x, y):=\frac{1}{2 \pi i} \int_{\alpha_{r}-i / \log y}^{\alpha_{r}+i / \log y} F_{r}(s, y) x^{s} \frac{\mathrm{d} s}{s}+O_{\varepsilon}\left(x^{\alpha_{r}} F\left(\alpha_{r}, y\right)\left(Y_{\varepsilon}^{-1}+H(u)^{-c}\right)\right),
$$

où $H(u):=\mathrm{e}^{u /(\log (u+1))^{2}}$ et $c$ est une constante positive absolue convenable.

Posons $T_{0}:=\bar{u}^{2 / 3} /(u \log y)$. Un nouvel emploi de $(5 \cdot 30)$, permet de restreindre l'intervalle d'intégration dans $(5 \cdot 31)$ à $\left[-T_{0}, T_{0}\right]$ au prix d'un terme d'erreur convenable. Lorsque $|\tau| \leqslant T_{0}$, nous effectuons un développement limité à l'ordre 3 de la fonction $F_{r}\left(\alpha_{r}+i \tau, y\right)$. Cela conduit à la formule

$$
\frac{1}{2 \pi i} \int_{\alpha_{r}-i / \log y}^{\alpha_{r}+i / \log y} F_{r}(s, y) x^{s} \frac{\mathrm{d} s}{s}=\frac{x^{\alpha_{r}} F\left(\alpha_{r}, y\right)}{\alpha_{r} \sqrt{2 \pi \sigma_{2, r}}}\left\{1+O\left(\frac{1}{\bar{u}}\right)\right\},
$$

dont $(5 \cdot 27)$ découle immédiatement.

Remarque. Notons également que ce qui précède implique l'estimation

$$
\Psi_{r}(x, y) \asymp \frac{x^{\alpha_{r}} F\left(\alpha_{r}, y\right)}{\sqrt{\pi(y)}} \quad(2 \leqslant y \leqslant \log x) .
$$

Remerciements. Les auteurs tiennent à exprimer ici leur chaleureuse gratitude au rapporteur, pour de pertinentes remarques et de judicieuses suggestions. 


\section{Bibliographie}

[1] R. de la Bretèche \& G. Tenenbaum, Propriétés statistiques des entiers friables, Ramanujan J. 9 (2005), 139-202.

[2] R. de la Bretèche \& G. Tenenbaum, Entiers friables : inégalité de Turán-Kubilius et applications, Invent. Math. 159 (2005), 531-588.

[3] R. de la Bretèche \& G. Tenenbaum, On the friable Turán-Kubilius inequality, in : Anal. Probab. Methods Number Theory, E. Manstavičius et al. (eds), TEV Vilnius 2012, 259-265.

[4] R. de la Bretèche \& G. Tenenbaum, Sur l'inégalité de Turán-Kubilius friable, J. London Math. Soc. (2) 93, no 1 (2016), 175-193. .

[5] N.G. de Bruijn, On the number of positive integers $\leqslant x$ and free of prime factors $>y$, Nederl. Akad. Wetensch. Proc. Ser. A 54 (1951), 50-60=Indag. Math 13 (1951), 50-60.

[6] N.G. de Bruijn, On the number of positive integers $\leqslant x$ and free of prime factors $>y$, II, Nederl. Akad. Wetensch. Proc. Ser. A 69 (1966), 239-247.

[7] J.-M. De Koninck, A. Granville \& F. Luca (eds.) Anatomy of integers, Papers from the CRM Workshop held at the Université de Montréal, Montréal, QC, March 13-17, 2006, CRM Proceedings \& Lecture Notes, 46, American Mathematical Society, Providence, RI, 2008. viii+297 pp.

[8] V. Ennola, On numbers with small prime divisors. Ann. Acad. Sci. Fenn., Ser. A I 440 1969, 3-16.

[9] A. Granville, Smooth numbers : computational number theory and beyond, in : Algorithmic number theory : lattices, number fields, curves and cryptography, Math. Sci. Res. Inst. Publ. 44, Cambridge Univ. Press, Cambridge, 2008.

[10] G. Hanrot, B. Martin \& G. Tenenbaum, Constantes de Turán-Kubilius friables : étude numérique, Exp. Math. Experiment. Math. 19, $\mathbf{n}^{\circ} 3$ (2010), 345-361.

[11] A. Hildebrand, Integers free of large prime factors and the Riemann Hypothesis, Mathematika 31 (1984), 258-271.

[12] A. Hildebrand, On the number of positive integers $\leqslant x$ and free of prime factors $>y, J$. Number Theory 22 (1986), 289-307.

[13] A. Hildebrand \& G. Tenenbaum, On integers free of large prime factors, Trans. Amer. Math. Soc. 296 (1986), 265-290.

[14] A. Hildebrand \& G. Tenenbaum, Integers without large prime factors, J. Théorie des Nombres de Bordeaux 5 (1993), 411-484.

[15] B. Martin \& G. Tenenbaum, Sur l'inégalité de Turán-Kubilius friable, J. reine angew. Math. 647 (2010), 175-234.

[16] H.L. Montgomery, Ten lectures on the interface between analytic number theory and harmonic analysis, CBMS Regional Conference Series in Mathematics, 84, Published for the Conference Board of the Mathematical Sciences, Washington, DC, by the American Mathematical Society, Providence, 1994, RI. xiv+220 pp.

[17] C. Pomerance, The role of smooth numbers in number-theoretic algorithms, in : Proceedings of the International Congress of Mathematicians, Vol. 1, 2 (Zürich, 1994), 411-422, Birkhäuser, Basel, 1995.

[18] O. Robert \& G. Tenenbaum, Sur la répartition du noyau d'un entier, Indag. Math. 24 (2013), 802-914.

[19] G. Tenenbaum, Introduction à la théorie analytique et probabiliste des nombres, quatrième édition, coll. Échelles, Belin, 2015, 592 pp. (English translation : Introduction to analytic and probabilistic number theory, Graduate Studies in Mathematics 163, Amer. Math. Soc. 2015).

Régis de la Bretèche

Université Paris Diderot-Paris 7

Sorbonne Paris Cité, UMR 7586

Institut de Mathématiques de Jussieu-PRG

Case 7012, F-75013 Paris

regis.delabreteche@imj-prg.fr
Gérald Tenenbaum

Institut Élie Cartan

Université de Lorraine BP 70239

F-54506 Vandœuvre-lès-Nancy Cedex

gerald.tenenbaum@univ-lorraine.fr 